\title{
Using eHealth to monitor idiopathic pulmonary fibrosis
}

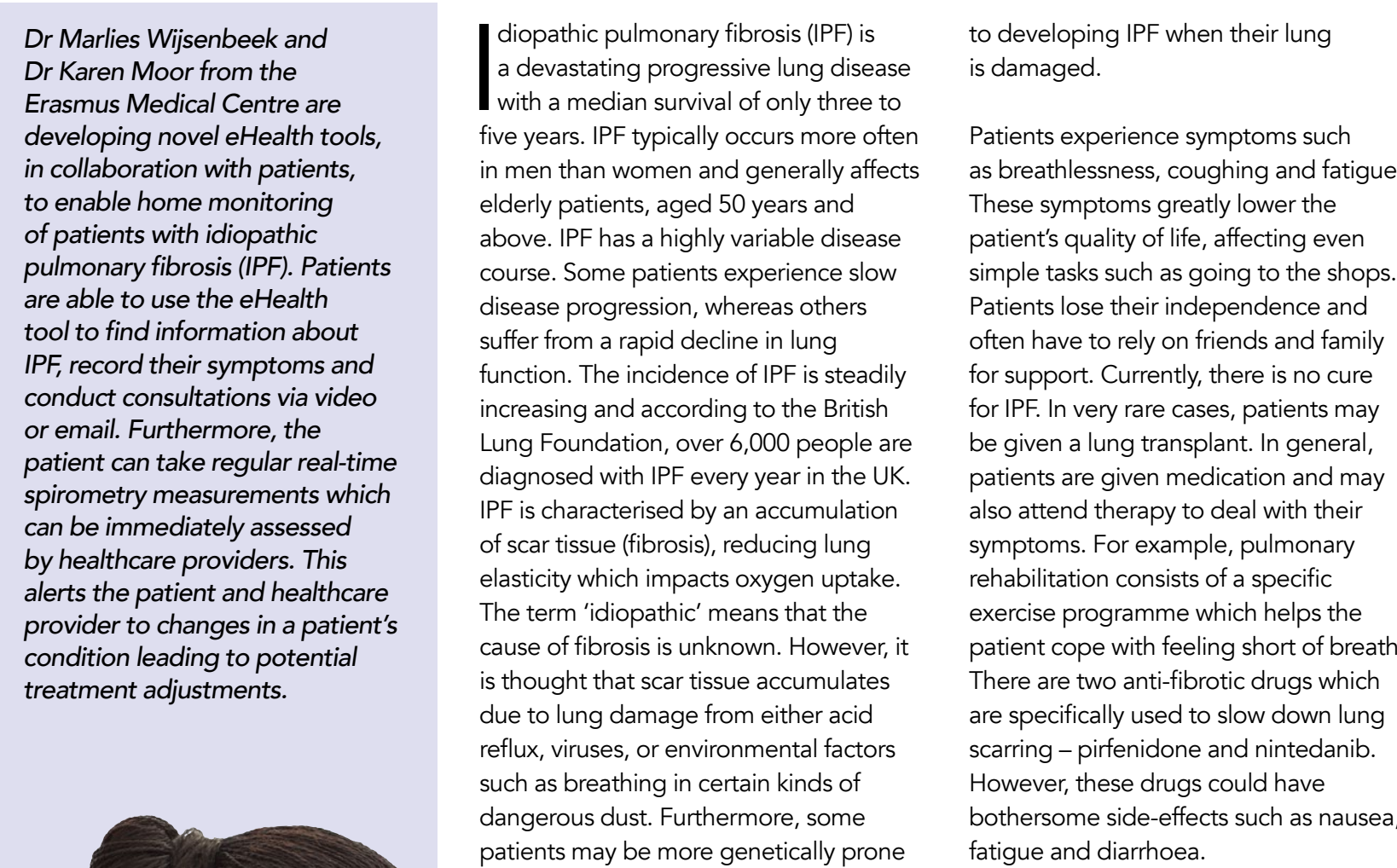

DrMarlies Wijsenbeek and Dr Karen Moor from the Erasmus Medical Centre are developing novel eHealth tools, in collaboration with patients, of patients with idiopathic pulmonary fibrosis (IPF). Patients are able to use the eHealth tool to find information about $I P F$, record their symptoms and conduct consultations via video or email. Furthermore, the patient can take regular real-time spirometry measurements which can be ithmediately assessed alerts the patient and health provider to changes in a patient's condition leading to potential treatment adjustments.

diopathic pulmonary fibrosis (IPF) is a devastating progressive lung disease with a median survival of only three to five years. IPF typically occurs more often
in men than women and generally affects in men than women and generally erove. IPF has a highly variab disese course. Some patients experience slow disease progression, whereas others suffer from a rapid decline in lung function. The incidence of IPF is steadily increasing and according to the British Lung Foundation, over 6,000 people are diagnosed with IPF every year in the UK. IPF is characterised by an accumulation of scar tissue (fibrosis), reducing lung elasticity which impacts oxygen uptake. The term 'idiopathic' means that the cause of fibrosis is unknown. However, it is thought that scar tissue accumulates due to lung damage from either acid reflux, viruses, or environmental factors such as breathing in certain kinds of patients may be more genetically prone

developing IPF when their lung is damaged.

Patients experience symptoms such Theseathlessness, coughing and fatigue patient's quality of life, affecting simple tasks such as going to the shops. Patients lose their independence and often have to rely on friends and family for support. Currently, there is no cure for IPF. In very rare cases, patients may be given a lung transplant. In general, patients are given medication and may also attend therapy to deal with their symptoms. For example, pulmonary rehabilitation consists of a specific exercise programme which helps the patient cope with feeling short of breath. There are two anti-fibrotic drugs which are specifically used to slow down lung However, these drugs cou Hower, hese dnugs could have fatigue and diarrhoea.

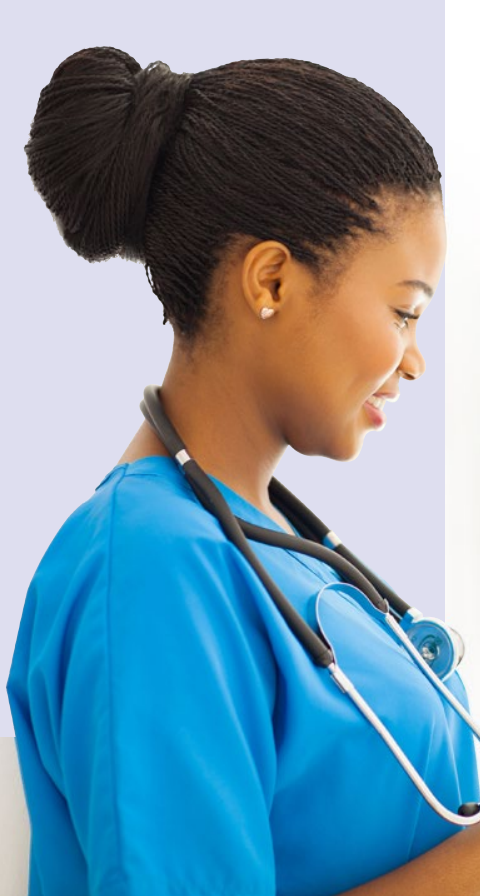

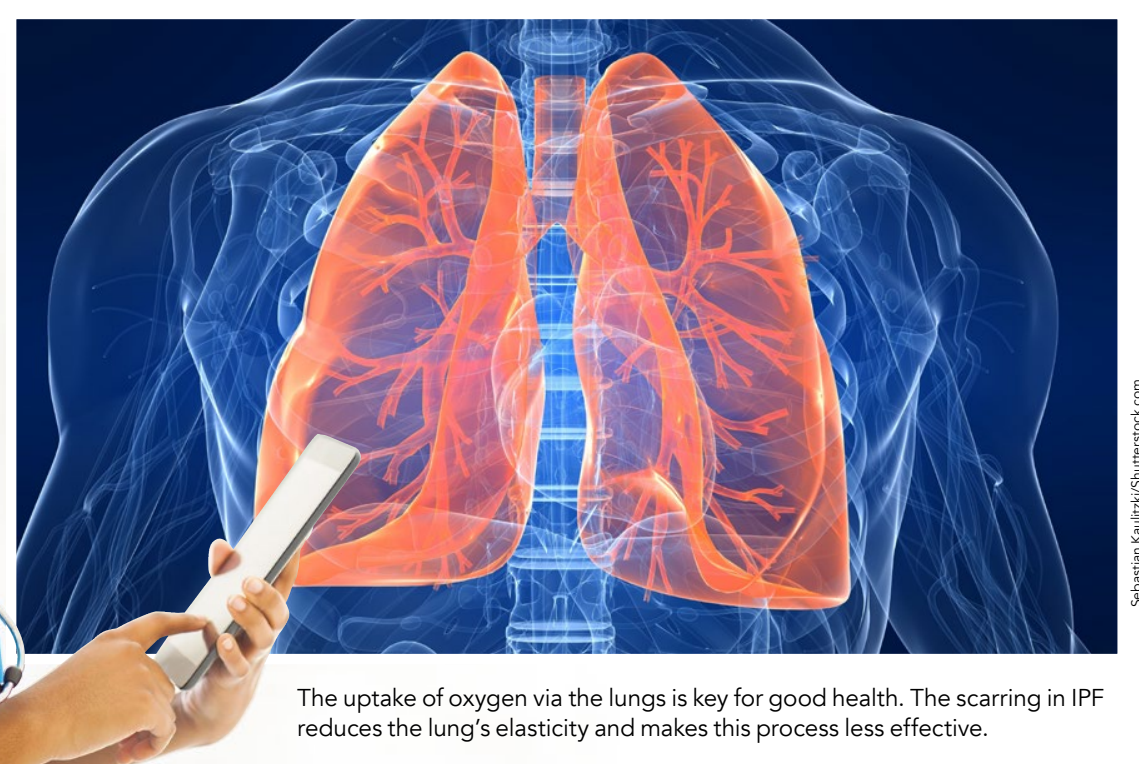
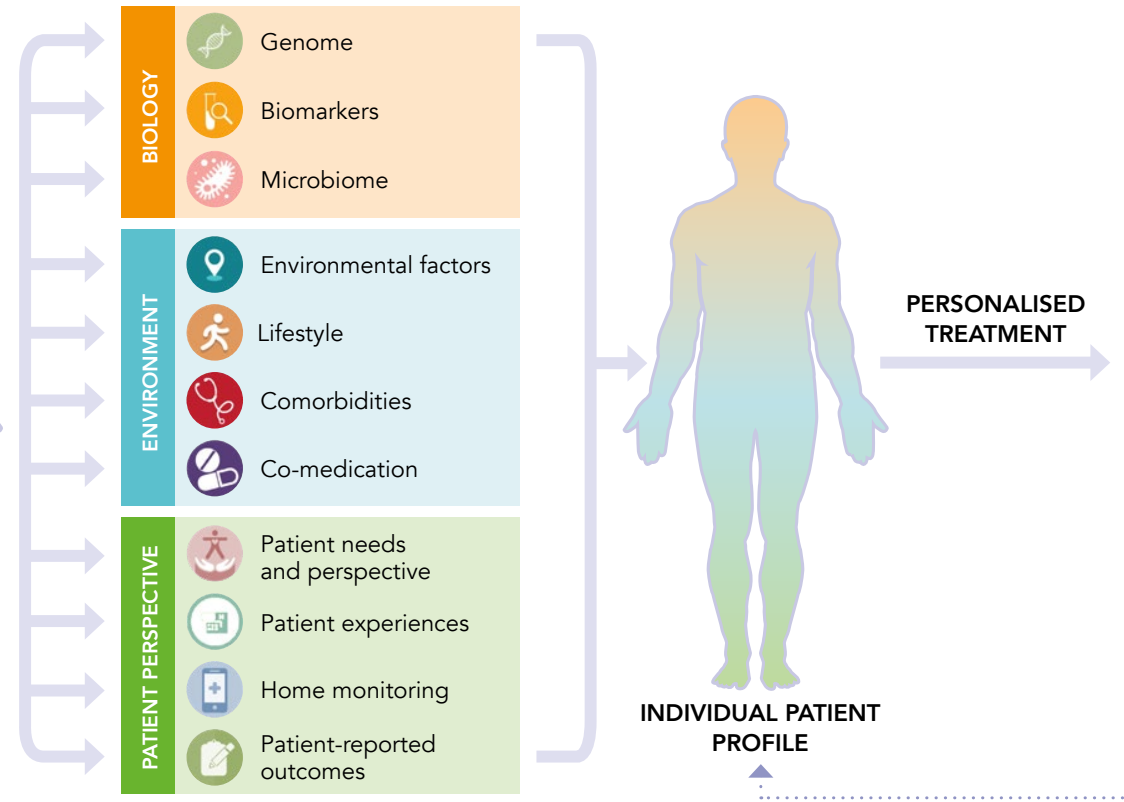

MONITORING ON
NTIERVENTION - Patient-reported Physiological Biology (ie. biomarkers, This diagram illustrates the numerous elements involved
as well as the reassessments and adjustments needed.

A personalised approach to IPF requires frequent monitoring due to the highly variable nature of IPF and the variation in response to therapy by different patients. DrWijsenbeek and Dr Moor from the Erasmus Medical Centre were

However, Dr Wijsenbeek and Dr Moor believe that patient factors, such as Iifestyle, comorbiditities, preferences and experiences should also play a engagement is also an important aspect. For example, it is vital to assess the

TREATMENT ADJUSTED

allowing for frequent home monitoring evaluation of treatment response and potential treatment adjustments, In fact, a previous study in IPF showed that home-based measurements predicted disease decline better than less frequent hospital-based measurements of lung tools to monitor the patient more
frequently athome eHealth involves the use of information home monitoring
could be invaluable with a low burden and communication technologies to for IPF patients as They aimed to exchange health-related data between to attend frequent this could improve the patient and healthcare provider. hospital visits due to reduced motility patient quality and breathles

\section{PERSONALISED MEDICINE} Lately, there has been increasing interest treat and monitor disease in IPF. Until now, personalised medicine has mainly focused on understanding the molecular mechanisms underpinning IPF. Little is understood about the influence of interactions between environmental, molecular and genetic mechanisms and how biological factors affect disease progression and influence the effectiveness of different treatment options. Improving our knowledge of these mechanisms could lead to the identification of specific biomarkers which could be used
targeted therapy. perspectives of patients before treatment. may show beneficial results at medica level. However for some individuals, certain drugs may prove ineffective the side effects may outweigh the benefits of treatment. Currently, over-use and under-use of medication is fairly common in IPF. However, this may not be the case if the patient's preferences are considered. These preferences may alter as the disease progresses, so personalised treatment plans must be regularly evaluated.

DrWijsenbeek and Dr Moor recognise the importance of personalising treatm for IPF, taking into consideration the patient's perspective, physiology and contribute to persolised adining
As a result, the team developed an ward-winning eHealth tool for patients health and become actively involved in managing their disease.

SHEALTH AND HOME MONITORING ealth involves the use of information d communication technologies to (x) ( D in collaboration with patients. This innovative eHealth tool won two Dutch prizes - the Patient Participation Prize prize in 2016. IPF Onlin has prize in 2016. IPF Online has many atures for patients to hils inclualing 
econsultations and video consultations information about videdication and to fill in their symptoms and possible sideeffects they may be experiencing and other patient-reported outcomes, such as quality-of-life questionnaires. Patientreported outcomes are reports of the patient's quality of life, health or function status that come directly from the patient without, for example, interpretation by clinicians.

IPF Online is integrated with real-time a spirometer to measure how much air is breathed out in one forced breath (forced vital capacity or FVC). Interestingly, the team believed that a major challenge many elderly people could possib hesitant using onle cold possibly be the majority of IPF patients who were asked to participate wanted to use IPF Online. By using IPF Online, the patient can gain insight into their own in the management of their disease. The team performed multiple studies to determine the feasibility of the home patient satisfaction

\section{PILOT STUDY}

the Erasmus involved in the pilot wireless spirometry. This is a simple test used to monitor lung conditions using health condition and become involved

study with home spirometry. Patients month using a and online patient-reported outcomes at baseline and after four weeks. The spirometry data is transmitted real-time and is directly available for analysis they start the programme . unthermo some patients had no internet access To tackle this issue, these patients could be supplied with a 4G SIM card to guarante internet access. Patients who had never used the internet before were able to

IPF patients found real-time spirometry very useful and would recommend it to others.

by both patients and healthcare providers. use the tablet with ease and perform Additionally, automated email alerts are spirometry due to the simple design. sent if the patient reports troublesome_-Additionally, some patients may not comply with taking measurements daily. $>10 \%$ for three consecutive days.

Overall, the results revealed that home spirometry highly correlated with hospita is a reliable test. Furthermore, $80 \%$ of patients considered daily spirometny easy and $90 \%$ thought that the process was not burdensome at all. All patients found realtime spirometry very useful and would recommend it to other IPF sufferers.

Patients and hospital staff in the study group did identify several potential issues with home monitoring and spirometry and recommendations were made. For example, a handheld spirometer may be difficult for patients to use. Therefore

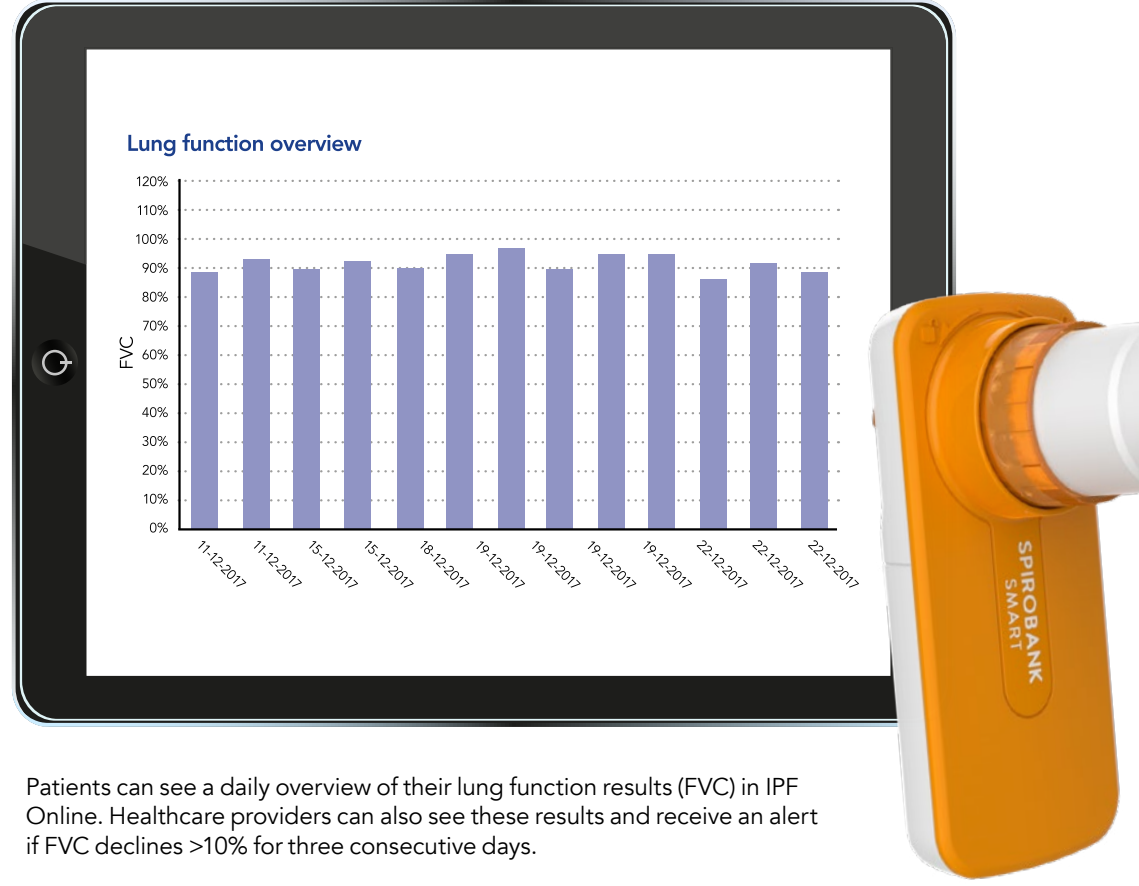
these situations, patients could be sent mail reminders.

A limitation of the pilot study is that it was performed at a single centre with a elatively small sample size. Although this was effective at highlighting reliability, patient satisfaction and identifying potential barriers, a larger scale, multiit improves patients' quality of life in the long term. The study team is currently addressing this by performing a multicentre randomised clinical trial with three other centres in the Netherlands. The team is following patients for six months to investigate whether the home monitoring programme/eHealth tool improves quality

\section{FUTURE PERSPECTIVES}

DrWijsenbeek and Dr Moor, in

collaboration with patients and healthcar that enables personalised individuallytailored therapy. Patients can understand condition and have
managing their own disease. Furthermo by performing real-time spirometry measurements, which are immediately

providers, changes in condition are quickly identified and the right treatment can be given. eHealth tools could revolutionise how we treat chronic, long-term conditions. In the future, eHealth tools could be in grated in clicical practice.

\section{Behind the Research}

Dr Marlies

Wijsenbeek

\section{Dr Karen Moor}

E: m.wijsenbeek-lourens@erasmusmc.nl E: c.moor@erasmusmc.nl T: +31650031750 W: www.ipfonline.n.

\section{Research Objectives}

Drs Wijsenbeek and Moor evaluated a new home monitoring programme with real-time wireless home spirometry in idiopathic pulmonary fibrosis.

\section{Detail}

Erasmus Medical Center Rotterdam Department of Respiratory diseas Dr. Molewaterplein 40 3015 GD

Bio

Marlies Wijsenbeek is a pulmonary Erasmus MC in Rotterdam. She is chair of the Erasmus MC multidisciplinary interstitial lung disease center (ILD) centre

Pneumonia Group of the European
Respiratory Society and the Dutch
National ILD section, and board member
of the Netherlands Respiratory Society.
Her research interests include eHealth
and other patient-centred outcome
measures in interstitial lung diseases, and
new therapies in IPF and sarcoidosis.
Karen Moor is a PhD candidate at the
Respiratory Department of the Erasmus
MC in Rotterdam. The aim of her research
is to improve clinical outcome measures

and quality of life for patients with IPF, with a special focus on the developmen and use of eHealth tools.

Funding

, Boehringer Ingelheim Erasmus MC Thorax Foundation

\section{Collaborators}

domised controlled trial hospital and OLVG in the Netherlands.

\section{References}

Moor, C.C., Heukels, P., Kool, M. and Wijsenbeek, M.S., 2017. Integrating patient perspectives into personalized medicine in

Moor, C.C., van Manen, M.J., Tak, N.C., van Noort, E. and Wijsenbeek, M.S., 2018. Development and feasibility of an eHealth tool for idiopathic pulmonary fibrosis. European Respiratory Journal,51(3), p. 1702508

Moor, C.C., Wapenaar, M., Miedema, J.R., Geelhoed, J.J.M., Chandoesing, P.P. and Wijsenbeek, M.S., 2018. A home monitoring program including real-time wireless home spirometry in idiopathic pulmonary fibrosis: a pilot study on experiences and barriers. Respiratory research, 19(1), p.105.

Idiopathic Pulmonary Fibrosis. British Lung Foundation. Available at: https://www.blf.org.uk/support-for-you/ idiopathic-pulmonary-fibrosis-ipf [Accessed 12/02/2019]

\section{Personal Response} How could eHealth tools, such as IPF Online,
improve patients' quality of life in the long term? II eHealth tools enable frequent monitoring at home at a low burden for patients. This is especially important
in a chronic progressive disease such as IPF which has a huge impact on patient quality of life and a high symptom
burden. Our home monitoring programme IPF Online burden. Our home monitoring programme IPF Online has the potential to improve quality of life through pat
engagement, better medication use, low-threshold engagenicht, bettermedication use, low-thresh and earlier detection of disease deterioration.

\section{Erasmus MC}

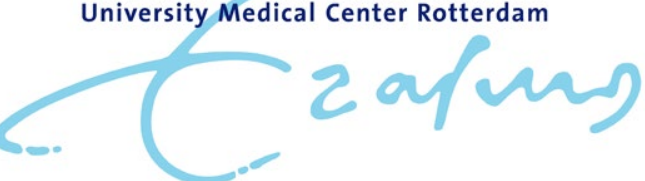

